\title{
EL USO DIDÁCTICO Y METODOLÓGICO DE LAS TABLETAS DIGITALES EN AULAS DE EDUCACIÓN PRIMARIAY SECUNDARIA DE CATALUÑA
}

\section{THE DIDACTIC AND METHODOLOGICAL USE OF TABLETS IN CLASSROMS OF PRIMARY AND SECONDARY EDUCATION IN CATALONIA}

\author{
Laura Fernández Rodrigo \\ edu.laurafernandez@gmail.com
}

Universitat de Lleida. Facultat d'Educació, Psicologia i Treball Social. Departamento de Pedagogía y Psicología. Avenida de l'Estudi General nº4, 25001, Lleida (España)

En esta investigación se describe y se diferencia el uso didáctico y metodológico de las tabletas digitales entre los centros de educación primaria y los de secundaria de Cataluña. Es un estudio cualitativo en el que se realizan 14 entrevistas a docentes y alumnos de primaria y secundaria de los 5 centros participantes. Las conclusiones determinan que el uso didáctico y metodológico de las tabletas está condicionado por la finalidad con la que se adquieren, las estrategias pedagógicas que se imparten en el aula, los niveles educativos y la cantidad de dispositivos disponibles.

Palabras clave: tabletas digitales, m-learning, educación, TIC.

The investigation aims to describe and differentiate the didactic and methodological use of tablets between primary and secondary schools in Catalonia. It is research through a qualitative methodology, in which 14 interviews are carried out to teachers and students of primary and secondary schools of the 5 participating centres. The conclusions determine that the didactic and methodological use of tablets is influenced by which purpose are they acquired, the pedagogical strategies given at classrooms, the educational levels and the amount of available devices.

Keywords: tablets, m-learning, education, ICT. 


\section{Introducción.}

Según el Consell Escolar de Cataluña (2015), los dispositivos móviles poseen un gran potencial que tiene que ser aprovechado para fines educativos, ya que "posibilita la realización de ciertas operaciones específicas que de otra manera no sería posible de realizar [...], las hace más simples [...] y permite realizarlas desde cualquier lugar» (p. 8). El año 2013 la Generalitat de Cataluña inicia el programa mSchools. Es un programa pionero educativo destinado a los estudiantes de primaria y secundaria de Cataluña que promueve el uso de dispositivos móviles para aprovechar su potencialidad en el proceso de aprendizaje (MWCB, 2013). Desde esta iniciativa se reconocen las innovaciones didácticas con los dispositivos móviles, se propone realizar visitas escolares al Mobile World Centre y se motiva a los centros a realizar otras actividades, como el diseño de apps, para reducir la brecha digital en el aula. El objetivo es mejorar el rendimiento académico, reducir las tasas de abandono escolar y proporcionar a las próximas generaciones las habilidades digitales y el espíritu emprendedor que necesitarán para su futuro profesional.

El uso de las tabletas tiene que ir complementado y sustentado por una metodología correcta y un modelo didáctico a fin que los alumnos puedan lograr aprendizajes significativos. Según Cánovas (2014) «la educación tradicional será un fracaso si sólo se utilizan las TIC para reproducir las mismas formas y contenidos simplemente en un formato digital» (p. 7). Las iniciativas de $\mathrm{mSchools}$ se proponen tanto para educación primaria como para secundaria, aunque la implementación de la tableta puede ser distinta en ambos etapas dado las prácticas educativas con el uso de las TIC sí que varían en función de la etapa en la que se implementan las clases (Instituto de Evaluación y Asesoramiento Educativo, Neturity y Fundación Germán Sánchez Ruipérez, 2006). Por estos motivos, los objetivos de este estudio son:

1) Describir la explotación didáctica y metodológica de las tabletas digitales en las aulas.

2) Identificar las diferencias y similitudes del uso didáctico y metodológico de las tabletas digitales entre las aulas de educación primaria y secundaria.

\subsection{M-Learning y la brecha digital.}

El aprendizaje móvil o m-learning es la modalidad educativa que facilita la construcción del conocimiento, la resolución de problemas de aprendizaje y el desarrollo de destrezas o habilidades de forma autónoma y ubicua gracias a la medicación de dispositivos móviles (Brazuelo \& Gallego, 2011). Cada día la brecha digital - que es la separación entre personas que utilizan las TIC como parte rutinaria de la vida cotidiana y las que no tienen acceso a las mismas- es menos evidente dado que a través de los dispositivos móviles todo el mundo puede acceder al conocimiento de las TIC sin tener que realizar una gran inversión en comprar un ordenador (Serrano \& Martínez, 2003). En este punto, Cánovas (2014) y Pedró (2011) afirman la existencia de una nueva brecha digital, la que separa los «productores digitales» de los «consumidores digitales» y está relacionada con la desigualdad de los beneficios del acceso a la tecnología. En el primer grupo se sitúan las personas que utilizan Internet de forma productiva y creativa 
-transmitiendo ideas, creando propuestas y espacios, generando contenidos, etc.- $y$, en el segundo grupo, aquellas que utilizan las TIC para chatear, leer el diario, mirar movimientos bancarios, comprar, usar el correo electrónico, etc. Desde la escuela, delante de la precoz edad de inicio en el uso de las TIC, se tendría que promover una educación basada en la «actividad, la creatividad, la generación de contenidos, idea y movimientos que pueden llegar a transformar la educación y la sociedad en la que crecen» (Cánovas, 2014, p. 7), a fin de cumplir los objetivos educativos a través de las TIC y con las TIC.

\subsection{Funciones didácticas de las tabletas digitales.}

Como dispositivo, las tabletas digitales son herramientas con un alto grado de interactividad por su pantalla táctil y muy intuitivos, ya que no requieren una capacitación previa y se integran naturalmente a las capacidades que los infantes han desarrollado con el uso de dispositivos móviles en su vida cotidiana (Marés, 2012). Por su ligereza y tamaño, la tableta permite a los alumnos y docentes que se pueda llevar a la mano de un lugar a otro sin esfuerzos y la durabilidad de la batería facilita que no haga falta enchufarla mientras se utiliza. La tableta proporciona una docencia más eficaz, flexible y móvil (European Schoolnet, 2013). A partir de los cuatro ejes propuestos por Cataldi y Lage (2013), podemos contar con las tabletas digitales en el aula teniendo en cuenta las funciones didácticas que pueden aportar:

1) Son fuente de documentación e información. Se puede tener acceso a través de la red a libros electrónicos, a vídeos, música, imágenes y otros archivos multimedia. Los alumnos pueden utilizar la tableta como libro y, también, para buscar información en diferentes formatos: PDF's, noticias, libros, investigaciones, páginas web, blogs, libros, etc. Agudelo, González y Quiceno (2013) afirman que el uso de tabletas en las aulas puede fomentar el aprendizaje por descubrimiento. Las tabletas son un buen estímulo para despertar la curiosidad, motivación e interés de los alumnos y los maestros han de presentar herramientas para que el estudiante descubra por sí mismo sus aprendizajes.

2) Son laboratorios multimedia abiertos. Los alumnos pueden crear y producir contenido virtual. A través de la tablet se puede acceder a una gran variedad de apps con funciones muy específicas, por ejemplo, para crear pósters, cómics, editar vídeos y fotos, realizar collages, narraciones y dibujos digitales, etc. Los alumnos pueden gravarse y fotografiarse con la cámara para poder editar los archivos en las apps. También se puede contar con las herramientas de ofimática de Microsoft Office (o similares) a fin de realizar presentaciones y documentos para los trabajos del aula.

3) Poseen aplicaciones específicas creadas para el aprendizaje de áreas curriculares. Podemos descargar cantidad de aplicaciones de diferentes temáticas para que los alumnos jueguen mientras practican contenidos curriculares, como juegos de vocabulario, de cálculo mental, de música, etc. Además, el uso de la tableta como herramienta de juego para practicar contenidos curriculares, se basa en la acción y experimentación con adultos y compañeros, con el objetivo de acceder a los medios audiovisuales y tecnológicos, mejorar la coordinación óculo-manual y propiciar las 
capacidades cognitivas de atención, percepción y discriminación, entre otras (Morales, 2008).

4) Son herramientas de comunicación. Se puede acceder a los servicios de la web 2.0 para compartir trabajos y actividades elaboradas en el aula con el resto de compañeros, con los docentes y con las familias, como los blogs, las plataformas virtuales, y los sistemas de almacenamiento en la nube. El uso de wikis, blogs, redes sociales $u$ otras apps y webs 2.0 comunicativas, adaptadas al uso educativo, pueden reforzar el aprendizaje colaborativo porque ponen énfasis en la contribución y participación de los usuarios (Agudelo, González \& Quiceno, 2013).

\subsection{Aspectos pedagógicos y metodológicos.}

La incorporación de la tableta digital en el aula puede contribuir en la innovación, ya que puede resultar un «vehículo para la transformación educativa» (CITA \& CEO Miguel Delibes, 2011, p. 8). Aun así, según los mismos autores, las TIC por si solas no innovan ni la enseñanza ni el aprendizaje. Desde la escuela, el objetivo es que los alumnos desarrollen las competencias básicas y las tabletas - y las TIC en generaltienen que ayudar a lograrlo (Trujillo, 2014). No existe una metodología concreta para utilizar la tableta digital, ya que la tableta no debe ser el centro de la metodología (Trujillo, 2014). Aún así, se expone en la Tabla 1 diferentes estrategias con las que los dispositivos pueden contribuir positivamente en el aula para lograr un aprendizaje por competencias:

A partir del curso 2009-2010, el Departament d'Ensenyament de Catalunya pone en marcha el proyecto Educat 1x1, con el cual los alumnos de secundaria pueden adquirir un propio ordenador portátil subvencionado. Sin las infraestructuras adecuadas, sin una visión de la programación curricular, sin un replanteamiento del enfoque de la docencia y sin tener experiencias previas se dieron una serie de problemáticas que hicieron frenar este proyecto (Consell Superior d'Avaluació del Sistema Educatiu, 2010). Todos los centros adquirieron el dispositivo digital, pero no de la metodología correcta. «No es suficiente con la dotación del material tecnológico en el aula, sino que el que es sumamente importante es el cambio metodológico» (Andreu, 2011, p. 7) ya que en muchos centros se continuaba haciendo lo mismo que antes, pero con un portátil, y con muchos más problemas. El mismo autor afirma que las TIC no son una finalidad, sino que «son un recurso y un camino para lograr que los infantes tengan un buen nivel competencial» (p. 8).

A fin de lograr una correcta integración de las TIC en las aulas, Koehler y Mishra (2009) proponen el modelo TPACK (Technological Pedagogical Content Knowledge) que se compone por tres principales formas de conocimiento: el conocimiento del contenido, el conocimiento pedagógico y el conocimiento tecnológico.

Como se aprecia en la Figura 1, la unión de estos tres tipos de conocimiento da lugar al conocimiento tecnológico pedagógico del contenido, que es la base de la enseñanza eficaz con la tecnología. La tecnología se utiliza de manera constructiva para enseñar el contenido, ayuda a corregir problemas que se enfrontan los alumnos y se utiliza para construir sobre el conocimiento existente para desarrollar nuevas epistemologías o fortalecer las existentes (Koehler \& Mishra, 2009). La clave está en encontrar la manera en que estas tres formas de conocimiento se puedan 


\begin{tabular}{|c|c|c|c|}
\hline $\begin{array}{c}\text { TAREAS } \\
\text { INTEGRADAS }\end{array}$ & PROYECTOS & RINCONES & $\begin{array}{l}\text { SECUENCIAS } \\
\text { DIDÁCTICAS }\end{array}$ \\
\hline $\begin{array}{l}\text { Acciones orientadas a la } \\
\text { resolución de una } \\
\text { situación a través de la } \\
\text { movilización de los } \\
\text { recursos que dispone el } \\
\text { alumno (Martínez, } \\
2014 \text { ). }\end{array}$ & $\begin{array}{l}\text { Los alumnos plantean, } \\
\text { implementan y avalúan } \\
\text { proyectos que tienen } \\
\text { aplicación en la vida } \\
\text { cotidiana (Blank, 1997; } \\
\text { Dickinson et al., 1998; } \\
\text { Harwell, 1997). }\end{array}$ & $\begin{array}{l}\text { El aula se organiza en } \\
\text { rincones de trabajo, para } \\
\text { promover la libertad de } \\
\text { elección, el descubrimiento } \\
\text { y la investigación } \\
\text { (Rodríguez, 2011). }\end{array}$ & $\begin{array}{ll}\text { Conjunto de } & \text { actividades } \\
\text { ordenadas, estructuradas y } \\
\text { articuladas } & \text { para la } \\
\text { consecución } & \text { de unos } \\
\text { objetivos } & \text { educativos } \\
\text { (Zabala; 1995). } & \end{array}$ \\
\hline
\end{tabular}

Tabla 1. Estrategias y metodologías en el aula.

interrelacionar, (pedagogía, contenido y tecnología) creciendo y sustentándose la una con la otra para llegar a adquirir un conocimiento conjunto.

\section{Metodología.}

El presente estudio se enmarca dentro del paradigma interpretativo, ya que se pretende comprender e interpretar los fenómenos de una realidad construida socialmente (Reeves, 2006) y se desarrolla a través de una metodología cualitativa, que se refiere a aquellos procedimientos de investigación

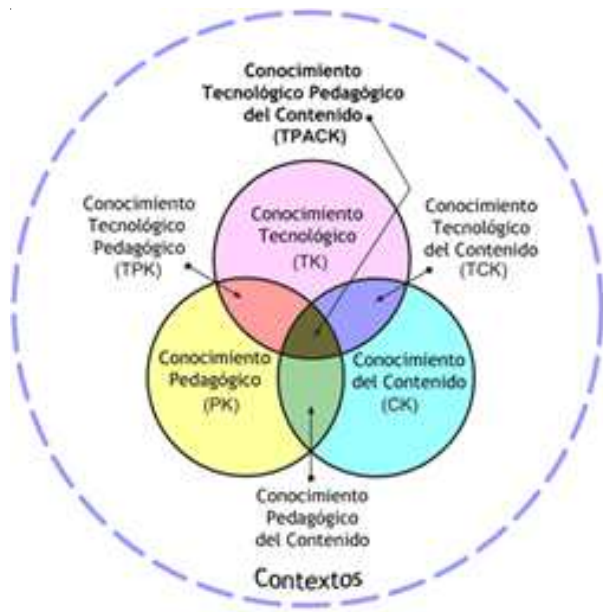

Figura 1. Modelo TPACK (Koehler, M. J. \& Mishra, P., 2009). que proporcionan datos descriptivos contextualizadas en el ámbito social dónde ocurren naturalmente (Bisquerra, 2009). La investigación se realiza a partir de un método etnográfico y microetnográfico, el cual consiste en la descripción y interpretación de una situación social concreta (Spradley, 1980). Se realizan un conjunto de entrevistas semiestructuradas a los participantes, que se elaboran a través de una pauta de preguntas ordenadas y redactadas por igual para todos los entrevistados, permitiendo una respuesta abierta y libre (Canales, 2006). A partir de los objetivos planteados al inicio del trabajo, se realizan tres guiones de entrevista con preguntas diferentes: el primero va dirigido a los equipos directivos, el segundo a los docentes de educación primaria y secundaria $\mathrm{y}$, el tercero, a los alumnos de las dos etapas educativas. Las preguntas están clasificadas por categorías, que son: aspectos metodológicos y estrategias pedagógicas, funcionalidades didácticas, competencias básicas, diversidad del alumnado y gestión de las tabletas en el aula. Los guiones han estado revisados por el director del trabajo y por el Departament d'Ensenyament de Cataluña. Se escogen centros educativos de modo intencionado, teniendo presente que tengan más de un curso escolar de experiencia en la implementación de tabletas digitales en 


\begin{tabular}{|c|c|c|c|c|c|c|}
\hline ETAPA EDUCATIVA & \multicolumn{2}{|c|}{ PRIMARIA } & \multirow{2}{*}{$\begin{array}{c}\text { SECUNDARIA } \\
3\end{array}$} & \multicolumn{2}{|c|}{$\begin{array}{l}\text { PRIMARIA Y } \\
\text { SECUNDARIA }\end{array}$} & \multirow[b]{2}{*}{$\begin{array}{c}\text { Total } \\
\text { entrevistados }\end{array}$} \\
\hline Centro & 1 & 2 & & 4 & 5 & \\
\hline Equipo directivo & 1 & 1 & 2 & 3 & 1 & 8 \\
\hline Equipo docente primaria & 4 & 1 & - & 2 & 1 & 8 \\
\hline Equipo docente secundaria & - & - & 3 & 2 & 2 & 7 \\
\hline Alumnos primaria & 3 & 2 & - & 2 & 2 & 9 \\
\hline Alumnos secundaria & - & - & 3 & 2 & 2 & 7 \\
\hline Total entrevistados & 8 & 4 & 8 & 11 & 8 & 39 \\
\hline
\end{tabular}

Tabla 2. Entrevistados en cada centro.

las aulas. De los 8 centros primeramente escogidos y contactados de Cataluña, 3 descartan la posibilidad de participar en la investigación. Por lo tanto, participan en el estudio un total de 5 centros educativos: 2 de educación primaria, 1 de secundaria y 2 escuelas-instituto. De cada centro participante, se entrevistan por separado a los siguientes grupos: equipo directivo, maestros, profesores, alumnos de primaria y alumnos de secundaria. Se han realizado un total de 19 entrevistas, en las cuales han participado 39 entrevistados distribuidos entre los siguientes colectivos y centros (Véase Tabla 2).

Las entrevistas, con la autorización de los centros, se registran con una grabadora de voz y se transcriben literalmente para analizar el contenido. Se extraen las ideas relevantes de cada entrevista a través de unidades de significado, que representan un fragmento de las aportaciones de los entrevistados. Finalmente, se realizan tablas comparativas clasificando las unidades de significado en las mismas categorías en las cuales se distribuían las preguntas iniciales de los guiones para las entrevistas.

\section{Resultados.}

A continuación, se presentan los resultados de las entrevistas realizadas a los equipos directivos, a los maestros y alumnos de educación primaria, y a los profesores y alumnos de secundaria. Los resultados se muestran a través de las Tablas 3, 4, 5, 6 y 7.

\subsection{Equipos directivos.}

Como se aprecia en los resultados del análisis expuestos en el Tabla 3, en secundaria se adquieren tabletas digitales a modo de 1x1, en cambio, en primaria, las tabletas son propiedad del centro educativo y se disponen de pocos dispositivos a compartir entre toda la etapa.

En las aulas de primaria, se emplean diferentes tipos de metodologías y estrategias en los diferentes centros y cursos. Los alumnos tienen que compartir la tableta y se introduce para utilizarla en una metodología de trabajo en grupos. En los cursos de ciclo inicial, la tableta se utiliza ocasionalmente para jugar con aplicaciones de contenidos 


\title{
USO DIDÁCTICO Y METODOLÓGICO DE LAS TABLETAS SEGÚN LOS EQUIPOS DIRECTIVOS
}

\author{
Metodología en el aula y estrategias \\ - Es diferente en cada área, curso y profesor.

\section{Funcionalidades didácticas} \\ - Jugar a apps de contenidos curriculares (en primaria) \\ - Producciones multimedia \\ - Compartir material en la red \\ - Creación y muestra de presentaciones \\ - Libro digital (en secundaria) \\ - Es multiuso. \\ - Las apps permiten realizar muchos tipos de tareas.
}

- Diversas estrategias en los diferentes centros y niveles: trabajo por tareas, secuencias didácticas, proyectos, rincones.

- Trabajo individual o en grupo dependiendo del docente y de la actividad

\section{Gestión y organización en el aula}

- Uso de la tableta: individual en un plan 1x1 (en secundaria), y en primaria se tiene que compartir.

- En primaria, se establece una tabla horaria para reservarlas o se distribuyen por las aulas.

- En primaria forma parte del material del centro.

- En secundaria, los alumnos se hacen responsable de su tableta.

\section{Competencias básicas \\ - Posibilita el trabajo cooperativo. \\ - La tableta motiva los alumnos.}

\section{Coordinación entre primaria y secundaria}

- Hay continuidad con el uso del dispositivo en secundaria.

- En secundaria se utiliza con más frecuencia, en primaria en ocasiones.

\section{Tabla 3. Resultados de las entrevistas a los equipos directivos.}

curriculares: «Tienen apps más originales, más interactivas, más adaptadas a ellos, por eso las pusimos en los cursos más pequeños» (Equipo directivo, centro 1). Tanto para los cursos de primaria y secundaria, la tableta es una herramienta para crear contenido digital, «para crear posters, para crear cómics, para crear vídeos, para editar» (Equipo directivo, centro 2).
En secundaria, la tableta es una herramienta personal y siempre se utiliza individualmente aunque se pueda trabajar en grupos. La metodología es distinta según el centro, la asignatura y el profesor. Se utiliza como libro digital, aunque también para crear contenido virtual, compartir información en la red, realizar trabajos colaborativos y hacer uso de una plataforma virtual, como Moodle. A excepción, 


\section{USO DIDÁCTICO Y METODOLÓGICO DE LAS TABLETAS SEGÚN LOS MAESTROS DE PRIMARIA}

\section{Metodología en el aula y estrategias}

- Se trabaja por proyectos: la tableta se utiliza para crear material y buscar información.

- Se trabaja por secuencias didácticas: la tableta se utiliza para crear material

- Rincones: se juega a apps de matemáticas, de lengua, de inglés, etc.

- Se fomenta el trabajo en grupo.

- Facilita la innovación educativa.

\section{Funcionalidades didácticas}

- Producciones multimedia: audio, imagen, video, cómic, pósters,...

- Uso de la cámara.

- Buscar información.

- Compartir contenido en la red.

- Se juega a apps para reforzar contenidos.

- Google Drive: para compartir archivos

- Realización de presentaciones

- A través de Internet, se tienen la información más actualizada que en los libros.

- Es multiuso.

\section{Gestión y organización en el aula}

- No se trabaja siempre con tabletas.

- Para determinadas actividades la reserva de las tabletas es permanente durante todo el curso.

- Muchas veces los alumnos tienen que compartir el dispositivo

\section{Competencias básicas}

- Es una herramienta que facilita el trabajo por competencias si la metodología también lo contempla.

- No se realiza ningún trabajo competencial sólo por el hecho de utilizar la tableta.

- Proporciona un aprendizaje más autónomo.

- La tableta motiva los alumnos.

\section{Atención a la diversidad}

- Es un soporte audiovisual.

- Está disponible inmediatamente, siempre está encendida.

- Es una herramienta de soporte.

- Hay apps para practicar con diferentes niveles.

Tabla 4. Resultados de las entrevistas a los/las maestros/as de educación primaria. 
el centro 5 también aplica un plan de $1 \times 1$ en ciclo superior y las tabletas se utilizan con las mismas finalidades que en secundaria.

\subsection{Maestros de educación primaria.}

Según los resultados del análisis expuesto en el Tabla 4, los centros disponen de un número reducido de tabletas para utilizar en educación primaria. El dispositivo en muchas ocasiones se tiene que compartir porque no se dispone de suficientes, por lo que se requiere que frecuentemente los alumnos trabajen en grupos, de manera cooperativa o por turnos. La metodología en el aula es diferente en cada centro educativo, curso o asignatura y la tableta digital se implementa como una herramienta que sirve para cumplir determinadas funciones en un contexto metodológico ya existente. No se ha cambiado la manera de trabajar en el aula para incorporar los nuevos dispositivos, sino que las actividades que se realizaban diariamente en el aula se han adaptado según las posibilidades didácticas que las tabletas ofrecen.

La tableta se utiliza con alumnos de ciclo inicial cuando se trabaja por rincones para practicar contenidos curriculares a través de apps de juegos de cálculo mental y de vocabulario, entre otras. Muchas de estas apps contienen diferentes niveles de dificultad, de manera que el ritmo depende de la habilidad y capacidad de cada alumno.

En los otros niveles de primaria, el dispositivo se utiliza con más frecuencia para realizar y editar vídeos, imágenes y sonidos, se pueden crear cuentos, cómics, se pueden compartir las producciones a la red, etc. Estas funcionalidades no se atribuyen a ninguna metodología de modo predeterminado, sino que según la actividad a realizar, se puede escoger la tableta o no, igual que se utiliza un lápiz, un bolígrafo u otro recurso. Aún así, hay estrategias en las cuales el uso productivo de la tableta es más eficaz porque la metodología requiere que los alumnos investiguen, creen y produzcan material, como en los proyectos que se realizan en los centros 2 y 4 , y las secuencias didácticas del centro 5. En estos casos, los alumnos trabajan por grupos cooperativos y utilizan la tableta para buscar información y crear contenido multimedia.

Como excepción, el centro 5 implementa un modelo de 1x1 a ciclo superior, por lo que la tableta es una herramienta que se utiliza con mucha más frecuencia que en los otros centros y se saca más partido a las funcionalidades que ofrece. Además de ser una herramienta de acceso a la información y de creación, utilizan el dispositivo para comunicarse entre los compañeros para realizar trabajos colaborativos en red y con los maestros para entregar las tareas y seguir el proceso de su evaluación.

\subsection{Profesores de educación secundaria.}

Como se muestra en la Tabla 5, los centros tenían experiencia con el uso de netbooks en el plan de 1x1 de la Generalitat para acceder a libros digitales y a la plataforma virtual. Con la incorporación de las tabletas, el centro 3 , mantiene una coexistencia entre libros de texto y libros digitales, el centro 4 utiliza libros digitales exclusivamente y el centro 5 opta por elaborar su propio material de aprendizaje. El uso de la tableta siempre es individual, ya que cada alumno tiene la suya, aunque la metodología de aula varía según el área y el profesor y se realizan más o menos trabajos por grupos o individualmente.

La tableta permite, además de acceder a los libros digitales y a la plataforma virtual, realizar otro tipo de tareas. Los centros de 


\section{USO DIDÁCTICO Y METODOLÓGICO DE LAS TABLETAS SEGÚN LOS PROFESORES DE} SECUNDARIA

\section{Metodología en el aula}

- El uso de las tabletas es individual

- Se realizan tantos trabajos individuales como en grupo.

- Se fomenta el trabajo diario y la evaluación continuada.

- Se utiliza el libro de texto y/o en formato digital.

- Se trabaja por secuencias didácticas.

- Se trabaja por tareas.

- La metodología es diferente según la asignatura y el profesor que la imparte.

-Lo Importante en el aula es la metodología, la tableta es sólo una herramienta.

- No se ha abandonar el papel ni la libreta, ya que los alumnos deben aprender de todo y tener criterio para elegir.

\section{Funcionalidades didácticas}

- Se utiliza como libro digital, igual que con los anteriores netbooks.

- Se realizan producciones multimedia: vídeos, audios, podcasts, ...

- Se utiliza para buscar información en la red

- Uso de Google Drive para compartir archivos internamente.

- Uso de plataformas virtuales para compartir archivos, realizar actividades, cuestionarios y exámenes y comunicar informaciones del centro.

- Herramientas de ofimática

- Uso del iPad con el Airplay para compartir la pantalla en el proyector y realizar presentaciones.

- Herramientas para el trabajo colaborativo

- Uso en las salidas y excursiones

- Se pueden disponer de muchos tipos de apps.

- Tiene más prestaciones audiovisuales y más ventajas funcionales que un ordenador.

- A través de plataformas y herramientas de feedback, pueden tener las evaluaciones de inmediato.

- Los alumnos se conocen el funcionamiento de la tableta y pueden buscar nuevas apps fácilmente.

Tabla 5. Resultados de las entrevistas a los/las profesores/as de educación secundaria.

ESO participantes utilizan la tableta para realizar las mismas tareas que se podían hacer con ordenadores, como realizar trabajos de redacción, presentaciones, buscar en la red, gestionar la plataforma virtual, etc., de una manera más inmediata que con un portátil. Además de estas funcionalidades, la tableta permite realizar otro tipo de actividades que no se podía hacer con un ordenador, como grabar y compartir instantáneamente, acceder a un gran despliegue de apps de edición y creación multimedia y otros funciones muy concretas, compartir archivos inmediatamente entre los compañeros y profesores, compartir la pantalla con el proyector de manera inalámbrica (en el caso de iPad), etc.

El centro 5, a diferencia de los otros dos, no utiliza la tableta como libro digital, ya que 


\title{
USO DIDÁCTICO Y METODOLÓGICO DE LAS TABLETAS SEGÚN LOS PROFESORES DE
} SECUNDARIA

\author{
Competencias básicas \\ - Facilita el trabajo autónomo. \\ - Los alumnos aprenden a responsabilizarse. \\ - Los alumnos son autodidactas. \\ - Motiva a los alumnos. \\ - Permite realizar trabajos colaborativos. \\ - Permite crear el propio material.
}

\section{Gestión y organización en el aula}

- La tableta utiliza en el aula cuando lo señala el maestro.

- Las tabletas siempre están disponibles ya que cada alumno tiene la suya.

- La tableta se lleva a casa y se vuelve al día siguiente con la batería recargada.

\section{Atención a la diversidad}

- Se trabaja en grupos heterogéneos

- El ritmo de trabajo se puede adaptar y la tableta lo facilita.

- A través de la tableta todo tipo de alumnos pueden realizar la misma actividad.

- Los alumnos con NEE se sienten integrados porque utilizan la misma herramienta.

- La tableta es una herramienta de apoyo como otro tipo de material.

\section{Tabla 5. Resultados de las entrevistas a los/las profesores/as de educación secundaria.(continuación)}

los ordenadores portátiles tampoco se utilizaban para este fin:

Durante el año utilizamos libros digitales y aquel año nos sirvió al claustro por entender que no era el camino, es decir, no se trataba de sustituir un libro tradicional, que ya hace años que no utilizamos libros, sino que era utilizar una herramienta para lo que queríamos nosotros pedagógicamente, y entender que, por tanto, con los iPads no hemos tenido esta duda (Profesor 2, centro 5).

En este centro no se seguían libros de texto ya que no se creía en una pedagogía tradicional y se utilizaba el ordenador portátil como herramienta para crear conocimiento. De este modo, se incorporan las tabletas digitales como sustitución de los portátiles. El proyecto educativo de centro ya contemplaba este tipo de pedagogía y no se cambia la forma de enseñar para que entren en la escuela las tabletas digitales, sino que este dispositivo sirve para consolidar el mismo proyecto educativo.

\subsection{Alumnos de educación primaria.}

La tableta en todos los casos es una herramienta para crear y editar, y se utilizan apps más específicas según la tarea a realizar, como para crear cuentos y gravar-se. Como se observa en la Tabla 6, también se utiliza para buscar información en la red y a veces - 


\section{USO DIDÁCTICO Y METODOLÓGICO DE LAS TABLETAS SEGÚN LOS ALUMNOS DE PRIMARIA}

\section{Metodología en el aula}

-A veces se trabaja en grupos y otras veces individualmente

\section{Funcionalidades didácticas}

- Juegos de apps para reforzar contenidos (alumnos de CI).

- Búsqueda de información en la red.

- Leer lecturas.

- Apps para crear cuentos.

- Producciones multimedia.

- Herramientas de ofimática (alumnos de CS).

- Web 2.0 y apps para compartir archivos e información, como Google Drive (alumnos de CS).

- Los alumnos descubren recursos para utilizar la tableta.

\section{Gestión y organización en el aula}

- La tableta utiliza cuando lo indica el maestro.

- Se tiene que compartir o no dependiendo de las que haya disponibles y del número de alumnos.

- Se utiliza en determinadas asignaturas o actividades.

- Es mejor que ir al aula de informática.

Tabla 6. Resultados de las entrevistas realizadas a los alumnos de educación primaria.

sobre todo en los cursos más bajos de primaria- para jugar a apps con contenidos curriculares, como para practicar el cálculo mental o vocabulario.

La tableta no es el único dispositivo digital que se utiliza, ya que depende de que tarea se tenga que realizar es mejor un portátil o un ordenador, como indica un alumno: «Si el profesor nos dice 'tenemos que hacer un trabajo', no siempre utilizamos, todo el día, las tablets, sino, por ejemplo, tenemos que hacer alguna cosa con el ordenador» (Alumno 1, centro 5). Los alumnos del centro 1, a partir de tercero de primaria no utilizan tableta, pero utilizan ordenadores portátiles como herramienta habitual: «Nos envían deberes por el ordenador por Moodle, i también empezamos a $4^{\circ}$ con medio a hacer servir
Flipped Classroom» (Alumno 3, centro 1). Por tanto, se tiene que tener criterio -tanto por parte de los maestros en la organización de las actividades como para los propios alumnos- para escoger la herramienta de trabajo adecuada según la tarea a realizar.

La predisposición en todos los alumnos para trabajar con la tableta es muy positiva:

- «Sí, más contentos, más atentos, más eficaces porque nos gusta más» (Alumno 1, centro 4).

- «Es una manera más divertida de aprender» (Alumno 1, centro 2).

Los alumnos están motivados y les resulta fácil encontrar recursos para aprender a través de los dispositivos móviles. Valoran muy positivamente que no hayan de depender de la disponibilidad de la sala de informática, ya 


\section{USO DIDÁCTICO Y METODOLÓGICO DE LAS TABLETAS SEGÚN LOS ALUMNOS DE SECUNDARIA}

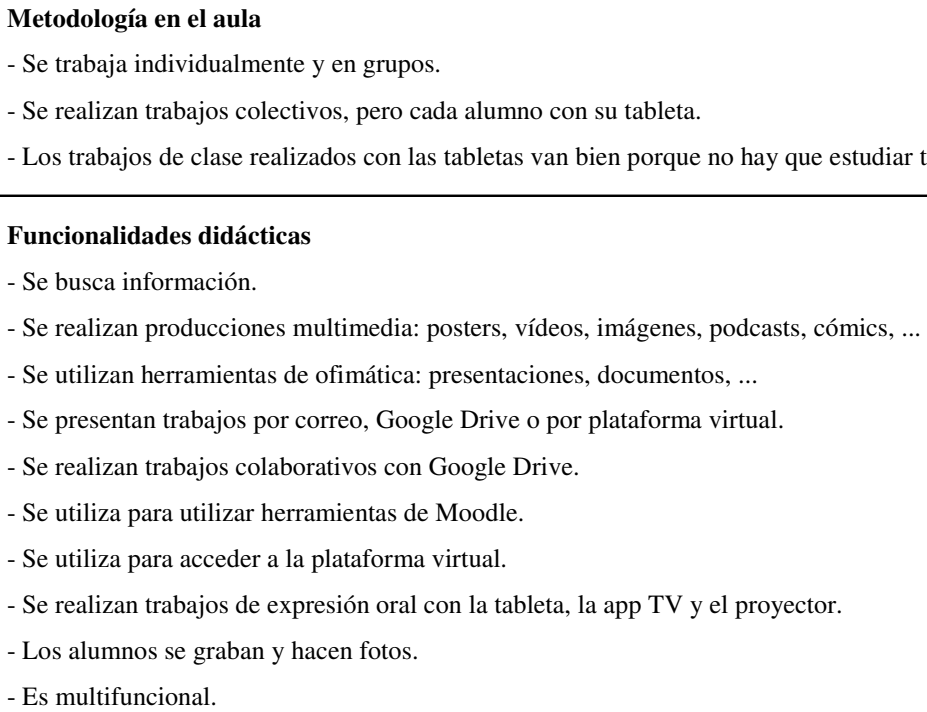

\section{Tabla 7. Resultados de las entrevistas realizadas a los alumnos de educación} secundaria.

que se tarda en cambiar de aula y encender los ordenadores.

\subsection{Alumnos de educación secundaria.}

La tableta, según los resultados expuestos en la Tabla 7, es de los alumnos y siempre está lista para usar, aunque es el profesor quien indica el momento y no se hace uso con la misma frecuencia en todas las asignaturas. Siempre se utiliza individualmente, aunque la manera de trabajar-dependiendo de la asignatura, de la actividad o del profesor- pueda ser por grupos o no. Los alumnos están satisfechos del modo en que se utiliza, ya que los ayuda a estudiar y a prepararse para los exámenes.

En los centros 3 y 4 la tableta es un complemento al libro (digital y/o en papel) y se hace servir para realizar trabajos que hace un tiempo realizaban con un portátil o en papel y para desarrollar actividades complementarias al temario de clase. La tableta, además, posibilita que se puedan 
realizar otro tipo de actividades que no se pueden hacer con otro dispositivo: «A veces hacemos trabajos y nos dejan [...] ir al patio $\mathrm{y}$ hacer fotos para ponerlas en los trabajos» (Alumno 2, centro 4).

En el centro 5 no se utilizan libros y los alumnos siempre elaboran su propio material de aprendizaje, en muchas asignaturas se trabaja por secuencias didácticas y es cuando más hacen servir el iPad. Se fomenta en trabajo cooperativo y colaborativo haciendo uso de Google Drive y la plataforma Clickedu. En todos los centros, con más o menos frecuencia, se realizan actividades creativas a través de la gran variedad de apps que se pueden descargar a las tabletas: «Estamos haciendo un trabajo a lengua que es un trabajo individual sobre unos efectos especiales, como se hacen y hemos hecho un cortometraje de tres minutos y con todo de efectos y está muy bien porqué es como un ovni que llega a la tierra y esto es más divertido y didáctico» (Alumno 2, centro 5).

\section{Discusión.}

Las diferencias del uso educativo de la tableta entre primaria y secundaria están condicionadas por el modelo de implementación, ya que en secundaria se implementa un modelo de $1 \times 1$-igual que en ciclo superior de uno de los centros- y en primaria los dispositivos son de la escuela y no se dispone de demasiados. El equipo educativo de primaria se ve obligado a utilizar la tableta en determinadas ocasiones y a menudo de forma compartida, por parejas o en pequeños grupos. La manera de trabajar con un modelo de 1x1 es diferente porque, aunque se trabaje en grupos, cada alumno tiene su propio dispositivo y lo utiliza individualmente. Los alumnos llevan la tableta de casa a la escuela cada día, como el estuche o una libreta, pueden seguir las actividades del aula desde cualquier lugar y momento. La tableta, en un entorno de 1x1, se utiliza para realizar trabajos de ofimática y como herramienta de comunicación entre alumnos y profesores, a través de apps de almacenamiento en la nube, plataformas virtuales y correo electrónico. Teniendo en cuenta el modelo TPACK, en secundaria, donde se implementa un modelo de 1x1, las tabletas digitales están mucho más integradas en las prácticas pedagógicas del aula que en educación primaria, dónde sólo se puede disponer de esta herramienta ocasionalmente.

Ante la variedad de estrategias pedagógicas que se implementan en las aulas, se tiene presente las funciones didácticas que posibilitan las tabletas y en cómo esta herramienta se puede utilizar para las actividades que se llevan a cabo en el aula. Delante de una nueva brecha digital (Cánovas, 2014; Pedró, 2011), los alumnos se posicionan como «productores digitales» cuando producen o editan un vídeo u otras actividades creativas que se llevan a cabo tanto en primaria como en secundaria o comparten sus producciones en la red. Por otro lado, se posicionan como «consumidores digitales» los alumnos de ciclo inicial que juegan a apps para practicar contenidos curriculares y los alumnos de secundaria que utilizan la tableta para acceder a los libros digitales.

No existe una metodología y una didáctica concreta y única para utilizar la tableta digital en el aula. Cada centro tiene su propio proyecto y las tabletas digitales se adquieren para diferentes finalidades educativas. Como afirma Trujillo (2014), la incorporación de las tabletas digitales debe servir para apoyar el mismo proyecto de centro para lograr un 
cambio innovador. Aún así, el uso de la tableta no siempre es innovador, ya que en ocasiones se utiliza para un modelo de aprendizaje tradicional, como para el acceso a libros digitales, para realizar apuntes y tenerlos organizados, etc. En la mayoría de los casos no significa un cambio pedagógico, sino funcional.

\section{Referencias bibliográficas.}

Agudelo, P.A., González, Y.V. \& Quiceno, Y.V. (2013). Metodología para implementar el uso de tabletas digitales para el conocimiento y la elaboración de historietas en clase de español del grado tercero de primaria del colegio Bryon Gavira bajo el enfoque socioconstructivista. Proyecto pedagógico mediatizado. Universidad tecnológica de Pereira. Facultad de Ciencias de la Educación. Licenciatura en comunicación e informática educativa. Disponible en http://repositorio.utp.edu.co/ d s pace/bitstrea m/1 1059/4276/1/ 371334A282M.pdf

Andreu, P. (9 de novembre de 2011). Conclusions generals del Programa Educat1x1. El meu nou espai [Blog]. Recuperado de http://blocs.xtec.cat/ pepandreu/2011/11/09/conclusions-generalsdel-programa-educat-1x1/

Bisquerra, R. (2009). Metodología de la investigación educativa ( $2^{\mathrm{a}} \mathrm{Ed}$.). Madrid: La Muralla, S.A.

Blank, W. (1997). Authentic instruction. In W.E. Blank \& S. Harwell (Eds.), Promising practices for connecting high school to the real world (pp. 15-21). Tampa, FL: University of South Florida.

Brazuelo, F. \& Gallego, D. (2011). Cursos de formación del profesorado en red del INTEF. Mobile Learning y Realidad
Aumentada. Recuperado de: http:// educalab.es/documents/10180/481804/ 17_Ficha_INTEF_MobileLearning_RA_2_2015.pdf/ 9fd79fc2-082c-4e48-a3bc-fb08cf818b85

Canales, M. (2006). Metodologías de Investigación Social ( $1^{\mathrm{a} E \mathrm{Ed}}$.). Santiago, Chile: Lom Ediciones.

Cánovas, G. (2014). Menores de edad y conectividad móvil en España: Tablets y Smartphones. Centro de Seguridad en Internet para los Menores en España: PROTEGELES, dependiente del Safer Internet Programa de la Comisión Europea. Recuperado de: http:// www.diainternetsegura.es/descargas/ estudio_movil_smartphones_tablets_v2c.pdf

Cataldi, Z. \& Lage, F.J. (2013). Entornos personalizados de aprendizaje (EPA) para dispositivos moìviles: situaciones de aprendizaje y evaluacioìn. EDMETIC. Revista de Educacioìn Mediaitica y TIC, 2 (1), 111135.

CITA \& CEO Miguel Delibes (2011). Proyecto Ebook y Educación. DEDOS: Tabletas digitales en el aula. Recuperado de http://www.territorioebook.com/includes/ d e s c arga.ph p ? i d = . / recursos / 1298988498_ProyectoDedos.pdf

Consell Escolar de Catalunya (2015). Les tecnologies mòbils als centres educatius. Generalitat de Catalunya. Recuperat de: http://consellescolarcat.gencat.cat/web/ . content/cons e $11_{\text {_ e s colar / }}$ actualitat_consell_escola/documents_pdf/ $\mathrm{s} \mathrm{t}$ a $\mathrm{t}$ i c $-\mathrm{f}$ i 1 e s / D o c 1 15_Tecnologies_mobils.pdf

Consell Superior d'Avaluació del Sistema Educatiu (2010). El projecte eduCAT 1x1. Informe d'avaluació. Una aproximació en la perspectiva de les directores $i$ directors de centres participants (curs 2009 - 2010). Barcelona: Generalitat de Catalunya. 
Departament d'Ensenyament. Recuperat de: http://csda.gencat.cat/web/.content/home/ consell_superior_d_avalua/pdf_i_altres/ static_file/ eduCAT1x1.pdf

Dickinson, K.P., Soukamneuth, S., Yu, H.C., Kimball, M., D’Amico, R., Perry, R.,... Curan, S.P. (1998). Providing educational services in the Summer Youth Employment and Training Program [Technical assistance guide]. Washington, DC: U.S.

European Schoolnet (2013). Introducción de las tabletas en los centros educativos: Evaluación del proyecto piloto para tabletas de Acer y European Schoolnet. Recuperado de: http://recursostic.educacion.es/eda/web/ $\mathrm{t}$ a b l e $\mathrm{t}$ a s / d o c u m e n t o s / TabletEvaluation_exec-summ_ES.pdf

Harwell, S. (1997). Project-based learning. In W.E. Blank \& S. Harwell (Eds.), Promising practices for connecting high school to the real world (pp.23-28). Tampa, FL: University of South Florida.

Instituto de Evaluación y Asesoramiento Educativo, Neturity y Fundación Germán Sánchez

Koehler, M.J. \& Mishra, P. (2009). What is technological pedagogical content knowledge?. Contemporary Issues in Technology and Teacher Education, 9 (1), 60-70.

Marés, L. (2012). Tablets en educación. Oportunidades y desafíos en políticas uno a uno. Relpe. OEI. Oficina Regional de Buenos Aires. Recuperado de: http://www.oei.es/ 70cd/Tabletseneducacion.pdf

Marquès, P. (2013) Las tabletas digitales en las aulas de clase: 4 modelos tecnológicos para facilitar su utilización [web]. Red Social DIM. Portal de las tabletas digitales y los libros de texto digitales. Recuperado de: http:/ /peremarques.net/tabletasaulas.htm
Martínez, E. (2014). Competencias básicas y tareas integradas en el aula. Avances en Supervisión Educativa, 22. Recuperado de: http://www.adide.org/revista/images/stories/ revista22/ase_22_c_04_martinez_lobato.pdf

Morales, A. (2008). El ordenador en educación infantil. Recuperado de: http:// www.csi-csif.es/andalucia/modules/ mod_ense/revista/pdf/Numero_25/ ANA_MORALES_GOMEZ01.pdf

MWCB (2013). El manual de l'Expert mSchools. Recuperado de: http:// experts.mobileworldcapital.com/files/expertsmschools-intro.pdf

Pedró, F. (2011). Tecnología y escuela: lo que funciona y por qué. Documeno básico. Fundación Santillana. Recuperado de: http:// www.fundacionsantillana.com/upload/ f i cheros/noticias/201111/ documento_bsico.pdf

Reeves, T.C. (2006). Design research from the technology perspective. In J. Van, K. Gravemeijer, S. Mckinney \& N. Nieveen (Eds.), Educational design research (pp. 86-109). London: Routledge.

Rodríguez, J. (2011). Los rincones de trabajo en el desarrollo de competencias básicas. Revista Docencia e Investigación, 21, 105130.

Ruipérez (2006). Las tecnologías de la información y de la comunicación en la educación. Informe sobre la implantación y el uso de las TIC en los centros docentes de educación primaria y secundaria (curso 2005-2006). Plan avanza. Ministerio de Educación y Ciencia. Recuperado de: http:// www.oei.es/tic/TICCD.pdf

Serrano, A. \& Martínez, E. (2003). La Brecha Digital: Mitos y Realidades. México: UABC.

Trujillo, F. (20 de febrero del 2014) Pon una tableta en tu aula... però que no sea de 
chocolate [Blog].Edu@contic. El uso de las TIC en las aulas. Recuperado de: http:// www.educacontic.es/blog/pon-una-tabletaen-tu-aula-pero-que-no-sea-de-chocolate

Spradley, J.P. (1980). Participant Observation. United States of America: Holt, Rinehart and Winston.

Zabala, A. (1995). La praìctica educativa. Coìmo ensenPar. Barcelona: Graó.

Fecha de recepción: 17-07-2015

Fecha de evaluación: 24-09-2015

Fecha de aceptación: 30-10-2015

Píxel-Bit. Revista de Medios y Educación. $N^{o} 48$ Enero 2016. ISSN: 1133-8482. e-ISSN: 2171-7966. doi: http://dx.doi.org/10.12795/pixelbit.2016.i48.01 